Rev. Adm. Saúde (On-line), São Paulo, v. 19, n. 76: e184, jul. - set. 2019, Epub 10 set. 2019 http://dx.doi.org/10.23973/ras.76.184

ARTIGO DE REVISÃO

\title{
Gestão da qualidade no processo de automação laboratorial
}

Quality management in the laboratory automation process

\section{Bruna Ricelli', Juliana Gimenez Amaral²}

1. Biomédica. Aluna de pós-graduação em gestão de saúde do Centro Universitário SENAC, São Paulo SP.

2. Enfermeira, doutora em patologia experimental. Professora do Centro Universitário SENAC, São Paulo SP.

\section{RESUMO}

A introdução da automação laboratorial nos últimos anos definiu um novo cenário para os laboratórios, que passaram a visar a eficiência e otimizar a qualificação e quantificação de falhas em todas as fases dos processos no laboratório clínico: pré-analítica, analítica e pós-analítica, bem como na implantação de medidas corretivas e preventivas. O objetivo deste estudo é apresentar uma revisão sobre a evolução da gestão da qualidade na área da saúde, em específico na área laboratorial e demonstrar como a tecnologia e automação laboratorial foram responsáveis pelo surgimento de indicadores e tendências em medicina laboratorial. Trata-se de uma revisão integrativa da literatura, onde se pesquisou nas bases de dados BIREME, LILACS e SciELO, com os descritores cadastrados no DeCS. Após cruzamento e exposição aos critérios de inclusão e exclusão, foram selecionados 11 trabalhos. Os resultados mostraram que a gestão da qualidade nos laboratórios ganhou destaque com a implantação da automação, pois surgiu tempo hábil para os colaboradores realizarem essa função, aumentando os níveis de segurança para os pacientes, minimizando-se os erros na fase analítica, promovendo maior velocidade na produção e aumento da produtividade no setor laboratorial. Todos os textos analisados nesse trabalho apontam para a eficácia e crescimento da gestão da qualidade no setor laboratorial. Foi possível constatar que $\mathrm{o}$ atendimento das expectativas dos clientes (pacientes $\mathrm{e}$ 
organização de saúde) é responsabilidade do setor de gestão em saúde, que necessitam realizar um bom planejamento dos projetos. Sendo assim, este setor tem extrema importância na implantação da automação laboratorial, pois é capaz de identificar e solucionar falhas no processo, a fim de trazer benefícios para a cadeia de saúde.

Palavras-chave: patologia clínica, automação laboratorial, gestão da qualidade e técnicas de laboratório clínico.

\begin{abstract}
The introduction of laboratory automation in recent years has set a new scenario for laboratories, which are now aiming at efficiency and optimizing fault qualification and quantification at all stages of clinical laboratory processes: preanalytical, analytical and post-analytical, as well as the implementation of corrective and preventive measures. The objective of this study is to present a review on the evolution of quality management in health, specifically in the laboratory area and to demonstrate how technology and laboratory automation were responsible for the emergence of indicators and trends in laboratory medicine. This is an integrative literature review, where we searched the databases BIREME, LILACS and SciELO, with the descriptors registered in DeCS. After crossing and exposure to the inclusion and exclusion criteria, 11 papers were selected. The results showed that quality management in the laboratories gained prominence with the implementation of automation, as it was time for employees to perform this function, increasing the safety levels for patients, minimizing errors in the analytical phase, promoting greater speed. in production and increased productivity in the laboratory sector. All texts analyzed in this paper point to the effectiveness and growth of quality management in the laboratory sector. It was found that meeting the expectations of clients (patients and health organization) is the responsibility of the health management sector, which need to carry out good project planning. Thus, this sector is extremely important in the implementation of laboratory automation, as it is able to identify and solve process failures in order to bring benefits to the health chain.
\end{abstract}

Keywords: clinical pathology, laboratory automation, quality management and clinical laboratory techniques.

\title{
INTRODUÇÃO
}

A história da automação nos laboratórios clínicos está extremamente relacionada com a evolução dos conhecimentos em alquimia e química. A década de 1840 é considerada a origem da disciplina de química clínica que inicialmente era baseada em métodos analíticos clássicos, como colorimetria, 
absorção atômica, fotometria de chama, gasometria, potenciometria, amperometria e eletroforese, sendo necessários vários tipos de analisadores para atender às diferentes metodologias, trazendo enorme complexidade ao laboratório clínico em termos de pessoal, instalações e manutenção. $\mathrm{Na}$ história da automação laboratorial, tão importante quanto o desenvolvimento de sistemas analíticos automatizados foi a introdução, no final da década de 1950, pela Sigma Chemical Company, dos conjuntos diagnósticos, que consistem em reagentes pré-embalados e prontos para uso, em geral chamados de kits. ${ }^{(1,2)}$

A automação nos laboratórios clínicos tem seu auge na década de 1940, nos Estados Unidos, quando o número de exames laboratoriais começou a crescer em uma velocidade surpreendente, os profissionais que se disponibilizavam a trabalhar em grandes laboratórios de rotina estavam se tornando raros e os custos operacionais muito altos. Existiam pipetas semiautomáticas, colorímetros fotoelétricos e fotômetros, mas esses equipamentos apenas proporcionaram um ligeiro alívio na carga de trabalho. ${ }^{(2)}$

Em 1951, Leonard T. Skeggs Jr. (1918-2002), bioquímico norte-americano, estimava que em 10 anos os 35 mil exames realizados por mês no Veterans Administration Hospital, em Ohio, duplicariam e ele considerava que seria muito difícil manter a qualidade técnica na realização de um número tão grande de exames com os quatro analistas que trabalhavam em seu laboratório, então construiu o protótipo de um equipamento que revolucionaria a rotina laboratorial, o sistema denominado AutoAnalyzer, pelo qual Skeggs é considerado o fundador das análises clínicas automatizadas. Ele instituiu o conceito de análise por fluxo contínuo, uma forma de análise sequencial pela qual amostras de diferentes pacientes são transportadas por meio de uma mesma via, separadas por bolhas de ar ou algum líquido. Este foi o primeiro sistema completamente automatizado para a medida de ureia, glicose e cálcio, sendo uma associação de módulos nos quais eram desenvolvidas as diferentes etapas do processo analítico, projetadas de forma que a análise do sangue total se completasse sem nenhuma intervenção manual. ${ }^{(1,2)}$

Os primeiros autoanalisadores de fluxo contínuo podiam ser simples ou de multicanal, consistiam em uma membrana de diálise para filtrar o sangue total e produzir um filtrado livre de proteínas, tubos tygon, um gerador de bolhas de ar que eram utilizadas para separar amostras de diferentes pacientes, bobinas de mistura, uma cubeta de fluxo que permitia a leitura fotométrica e um registrador gráfico, em papel. Os modelos de multicanal permitiam a análise simultânea de vários analitos, sendo os mais comuns os de dois, seis e doze canais, com produtividade de aproximadamente 150 amostras por hora. Os sistemas eram não seletivos, sendo assim realizavam de um conjunto fixo de exames em todas as amostras, independentemente de terem sido solicitados ou não. Adotou-se então perfis, ou painéis de exames, conforme o número de exames realizados em cada amostra. Esse processo de automação, ainda que principiante, reduziu muito o tempo gasto para a realização de grandes rotinas, estimulou o uso de painéis de triagem, liberou o pessoal técnico para outras atividades e, principalmente, começou a modificar o perfil profissional do pessoal de laboratório, habilidade técnica manual e conhecimento bioquímico passaram a ter menor importância, sendo necessário conhecimento e habilidades para atender às demandas das máquinas. Os autoanalizadores de 
fluxo contínuo atingiram o auge em 1974, com o lançamento do Sequential Multiple Analyzer acoplado a um computador, o SMAC. ${ }^{(2)}$

Na década de 1990, surgiram os analisadores discretos ou analisadores de acesso aleatório, que são seletivos e executam diferentes testes em cada amostra, fazendo com que os analisadores de lote, como os autoanalizadores e analisadores centrífugos, que realizavam apenas um mesmo painel de exames em todas as amostras, começassem a ser descontinuados. Os analisadores de química clínica modernos usam sistemas discretos automatizados, permitindo uma combinação quase ilimitada de análises em um único instrumento, que combinam ensaios de química clínica e imunoensaios de rotina, possibilitando o uso de menor número de equipamentos e melhoria na eficiência operacional. Os analisadores são agora projetados para uma ampla gama de cargas de trabalho, que variam desde pequenas unidades de bancada a grandes modelos, de piso, com capacidades de produção baixa, média e alta. Tipicamente, o fabricante do analisador fornece os reagentes e os analisadores podem ser designados por sistema fechado, onde apenas os reagentes do fabricante do instrumento são adequados para utilização do analisador, ou sistema aberto, quando os reagentes de outros fornecedores podem ser utilizados. O desejável é que o analisador seja do tipo sistema aberto. ${ }^{(2,3)}$

Concomitantemente com o lançamento dos conjuntos diagnósticos para a automação laboratorial desenvolveu-se os sistemas de informação e de gestão de informações laboratoriais, na década de 1970 começaram a ser introduzidos esses sistemas de informações para o gerenciamento eletrônico de dados, fluxo de trabalho e como interfaces eletrônicas dos instrumentos. Inicialmente, foram criados para atender à necessidade de controlar os códigos de faturamento, mas ajudaram a automatizar as operações no laboratório, dispensando registros em papéis e possibilitando a captura de dados diretamente de equipamentos analíticos. ${ }^{(2,4)}$

A automação laboratorial total inclui as fases pré-analíticas, analíticas e pósanalíticas, caracterizada pela realização mecânica de vários processos, como recepção e triagem de amostras, destampamento dos tubos, centrifugação, fracionamento, entrega das amostras aos analisadores, retampamento dos tubos, armazenamento e registro das amostras, com capacidade de localizar e recuperar uma amostra para eventual diluição, repetição ou realização de teste complementar. Como vantagens temos, praticamente, a eliminação do erro humano no manuseio das amostras e na realização dos exames, redução das etapas de manipulação das amostras, como verificação de qualidade (hemólise/icterícia/lipemia), aumentando a segurança e produtividade dos profissionais, com redução e padronização do tempo de resposta. ${ }^{(5,6)}$

A história da qualidade tem início com a evolução industrial nos anos 1920, quando em decorrência da grande guerra mundial as indústrias necessitavam aumentar a produção de armamentos. Surge então a atividade de inspeção com finalidade de avaliar o produto final e separar os defeituosos, evitando sua comercialização. Nas décadas subsequentes, a evolução da qualidade tornouse mais evidenciada e a preocupação com a qualidade chega ao gerenciamento das empresas. Em 1950, W. Edwards Deming cria um novo 
conceito em qualidade denominado ciclo PDCA, cujas iniciais em inglês, significam plan, do, check, act, ou planejar, executar, verificar e atuar corretivamente. No ano seguinte, Joseph M. Juran publica a obra denominada Quality Control Handbook, onde contempla o controle de custos da qualidade, abordando os termos custo da não qualidade e retrabalho. Em 1956, A. Feigenbaum, propõe o conceito de controle total da qualidade, preconizando que a responsabilidade da qualidade do produto é de toda a organização e não somente do departamento de controle da qualidade. Atualmente, as exigências da qualidade são atributos essenciais à sobrevivência das organizações no mercado, com ênfase na satisfação do cliente. ${ }^{(4,6,7)}$

O setor laboratorial pode ser considerado como pioneiro na área médica a promover e introduzir os conceitos da qualidade. Nos anos de 1990, houve um consenso sobre os objetivos da qualidade e suas especificações no ambiente do laboratório clínico, assim definiu-se os conceitos de controle de qualidade, garantia da qualidade e gestão total da qualidade. A evolução tecnológica permitiu a implantação dos modernos conceitos da qualidade, sempre buscando uma melhoria contínua, o que exigiu então, uma análise minuciosa dos diferentes processos envolvidos na realização do exame laboratorial, incluindo aspectos técnicos, organizacionais e administrativos, além de identificar desvios e propor oportunidades de melhoria. ${ }^{(7)}$

As diversas etapas de execução de um exame laboratorial são divididas em três fases: pré-analítica, analítica e pós-analítica. Atualmente, os termos fase pré-analítica e pós-analítica foram introduzidos para definir etapas de execução de um exame que independem propriamente do laboratório, pois inclui-se aqui a seleção pelo clínico, de exames apropriados ao diagnóstico, coleta, transporte e recepção de amostras adequadas ao exame e interpretação do resultado pelo clínico. Sendo assim, o sistema de qualidade de um laboratório requer disciplina e organização em todas as etapas dos diferentes processos e nesse contexto os indicadores laboratoriais permitem avaliar a eficácia e a eficiência das diferentes etapas de execução do exame laboratorial. ${ }^{(5,6)}$

Serviços laboratoriais efetivos são a base dos sistemas de saúde modernos, as inovações científicas têm contribuído para melhorias notáveis na área laboratorial, porém os erros ainda persistem, a gestão de qualidade surge então como uma ferramenta essencial para identificar, analisar e solucionar esses erros. Sendo assim, o gestor tem como desafio ampliar os seus conhecimentos, envolver e treinar a equipe de profissionais, visando difundir os conceitos e a importância dos indicadores na gestão dos processos. ${ }^{(2,6)}$

Portanto, o objetivo deste trabalho é identificar na literatura textos que auxiliem a descrever o papel da gestão de qualidade no processo de automação laboratorial.

\section{METODOLOGIA}

Trata-se de uma revisão integrativa da literatura, método que permite uma abordagem ampla, possibilitando a inclusão de dados da literatura e 
incorporando a aplicabilidade dos resultados de estudos significativos na prática. ${ }^{(9)}$

Para busca dos estudos foram selecionadas as bases de dados: Biblioteca Regional de Medicina (BIREME), Literatura Latino-Americana e do Caribe em Ciências da Saúde (LILACS), Scientific Eletronic Library Online (SciELO). Utilizou-se os seguintes descritores: automação laboratorial, gestão da qualidade, gestão laboratorial e laboratório de análises clínicas, extraídos dos Descritores em Ciências da Saúde (DeCs). Como literatura adicional também foi consultada as regulamentações da área, guias e informações técnicas de órgãos oficiais. A pesquisa foi realizada entre novembro de 2018 e abril de 2019.

Os critérios de inclusão adotados para a busca e seleção das publicações foram os seguintes: artigos publicados em periódicos científicos nacionais e internacionais que abordassem a temática: automação laboratorial e gestão da qualidade, divulgados em língua portuguesa e inglesa, no período de 10 anos, disponibilizados na íntegra, diretamente pelo site da base de dados.

A busca foi realizada de maneira ordenada, classificando-se na primeira análise os artigos nas categorias: fora do período considerado, indisponíveis na forma completa, publicados em mais de uma base e os selecionados para segunda análise. Após, realizou-se a leitura do título e resumo de cada publicação a fim de verificar a paridade com a pergunta norteadora da pesquisa. Sendo assim, os que não abordavam o tema "automação laboratorial e gestão da qualidade" foram descartados na segunda análise.

Os resultados obtidos após esta análise foram exibidos em forma de fluxogramas e quadros, apresentados na seção de resultados e discussão do estudo.

\section{RESULTADOS E DISCUSSÃO}

Os resultados obtidos depois de busca nas bases de dados foram 2334 textos, sendo 2272 na BIREME, 41 na LILACS e 21 na SciELO, desses 2000 foram excluídos após a primeira análise e 334 encontraram-se dentro dos critérios de inclusão da primeira análise, após a leitura do título e resumo e uma segunda análise foram selecionados 42 artigos, esses foram lidos na integra e obteve-se ao final uma amostra de 11 textos, estas características são evidenciadas na Figura 1 


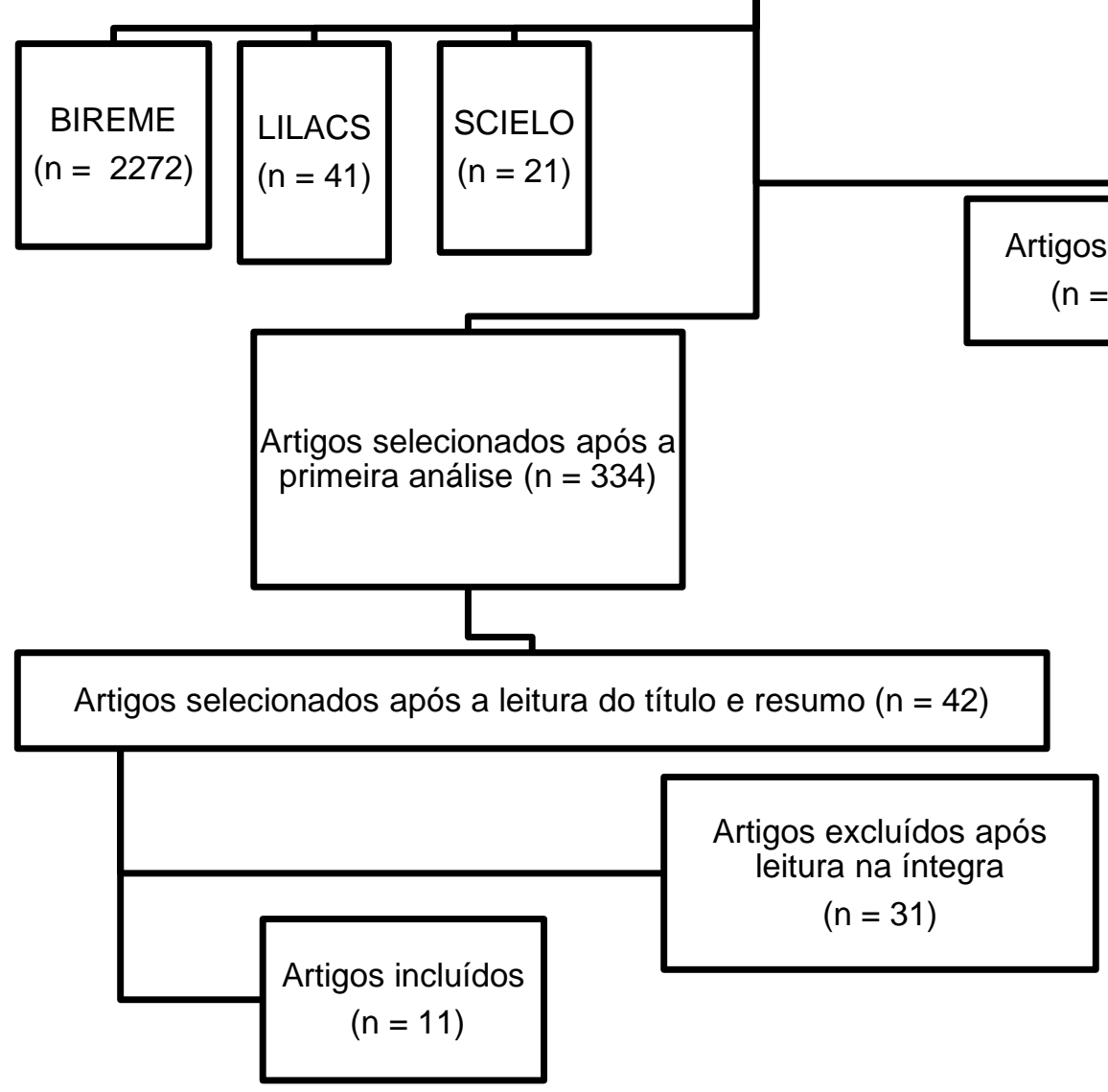

Figura 1. Processo de seleção dos estudos nas bases de dados Biblioteca Regional de Medicina (BIREME), Literatura Latino-Americana e do Caribe em Ciências da Saúde (LILACS), Scientific Eletronic Library Online (SciELO).

Após análise dos 11 textos selecionados de acordo com os critérios de inclusão, observa-se que todos são atuais demonstrando que a gestão laboratorial apesar de iniciada a muitos anos atrás continua sendo um assunto atual e que está sempre em atualização. De forma geral, os trabalhos encontrados na revisão têm como objetivo demonstrar e analisar métodos de gestão em saúde na prática, relacionando os aspectos positivos e negativos, o que pode ser observado no Quadro 1, pelos objetivos deles.

Quadro 1. Classificação dos textos encontrados segundo: título, autores, objetivo, ano de publicação e periódico.

\begin{tabular}{|l|l|l|l|l|}
\hline Título do artigo & Autores & Objetivo & $\begin{array}{l}\text { Ano de } \\
\text { publicação e } \\
\text { país }\end{array}$ & Periódico \\
\hline
\end{tabular}




\begin{tabular}{|c|c|c|c|c|}
\hline $\begin{array}{l}\text { A incorporação de } \\
\text { novas tecnologias nos } \\
\text { serviços de saúde: o } \\
\text { desafio da análise dos } \\
\text { fatores em jogo }\end{array}$ & Trindade, E. & $\begin{array}{l}\text { Demonstrar e analisar } \\
\text { as tecnologias para } \\
\text { saúde, seus avanços } \\
\text { bem como o modelo } \\
\text { de gestão adotado. }\end{array}$ & $\begin{array}{l}2008 \\
\text { Brasil }\end{array}$ & $\begin{array}{l}\text { Cad. Saúde } \\
\text { Pública }\end{array}$ \\
\hline $\begin{array}{l}\text { Gerenciamento de } \\
\text { projetos: o impacto do } \\
\text { uso dos indicadores de } \\
\text { desempenho no } \\
\text { resultado do projeto }\end{array}$ & $\begin{array}{l}\text { Filho, F.et } \\
\text { al }\end{array}$ & $\begin{array}{l}\text { Apresentar as } \\
\text { metodologias } \\
\text { utilizadas no } \\
\text { gerenciamento de } \\
\text { projetos e suas } \\
\text { métricas, } \\
\text { descrevendo a } \\
\text { análise estatística e o } \\
\text { uso de indicadores de } \\
\text { desempenho para } \\
\text { adoção de ações } \\
\text { corretivas. }\end{array}$ & $\begin{array}{l}2009 \\
\text { Brasil }\end{array}$ & $\begin{array}{l}\text { Produto e } \\
\text { Produção }\end{array}$ \\
\hline $\begin{array}{l}\text { Total lab automation } \\
\text { takes teamwork }\end{array}$ & $\begin{array}{l}\text { Peck- } \\
\text { Palmer, O. }\end{array}$ & $\begin{array}{l}\text { Estudo de caso, } \\
\text { através da automação } \\
\text { laboratorial total com } \\
\text { seleção de cinco } \\
\text { grupos } \\
\text { multidisciplinares para } \\
\text { implantação dos } \\
\text { objetivos estipulados } \\
\text { e posterior redução } \\
\text { de erros e melhora do } \\
\text { desempenho } \\
\text { laboratorial }\end{array}$ & $\begin{array}{l}2009 \\
\text { EUA }\end{array}$ & $\begin{array}{l}\text { Medical } \\
\text { Laboratory } \\
\text { Observer } \\
\text { (MLO) }\end{array}$ \\
\hline $\begin{array}{l}\text { A utilidade dos } \\
\text { indicadores de } \\
\text { qualidade no } \\
\text { gerenciamento de } \\
\text { laboratórios clínicos }\end{array}$ & Vieira et al. & $\begin{array}{l}\text { Demonstrar a } \\
\text { evolução da } \\
\text { qualidade na área da } \\
\text { saúde, especialmente } \\
\text { na área laboratorial e } \\
\text { o uso dos indicadores } \\
\text { laboratoriais na } \\
\text { otimização da } \\
\text { qualificação e } \\
\text { quantificação das } \\
\text { falhas nas diferentes } \\
\text { fases e áreas do } \\
\text { laboratório, auxiliando } \\
\text { na implantação de } \\
\text { medidas corretivas e } \\
\text { preventivas. }\end{array}$ & $\begin{array}{l}2011 \\
\text { Brasil }\end{array}$ & $\begin{array}{l}\text { J Bras Patol } \\
\text { Med Lab }\end{array}$ \\
\hline $\begin{array}{l}\text { Tendências em } \\
\text { medicina laboratorial }\end{array}$ & $\begin{array}{l}\text { Campana et } \\
\text { al. }\end{array}$ & $\begin{array}{l}\text { Descrever os fatores } \\
\text { que colaboram e são } \\
\text { fundamentais para o } \\
\text { crescimento da } \\
\text { medicina laboratorial, } \\
\text { discutindo e } \\
\text { apresentando as } \\
\text { principais tendências } \\
\text { que tem impacto } \\
\text { nesta área, } \\
\text { comprovando seus }\end{array}$ & $\begin{array}{l}2011 \\
\text { Brasil }\end{array}$ & $\begin{array}{l}\text { J Bras Patol } \\
\text { Med Lab }\end{array}$ \\
\hline
\end{tabular}




\begin{tabular}{|c|c|c|c|c|}
\hline \multicolumn{5}{|c|}{ benefícios. } \\
\hline $\begin{array}{l}\text { Conceitos de } \\
\text { automação na } \\
\text { medicina laboratorial: } \\
\text { revisão de literatura }\end{array}$ & $\begin{array}{l}\text { Campana, } \\
\text { C. Oplustil, } \\
\text { C }\end{array}$ & $\begin{array}{l}\text { Apresenta uma breve } \\
\text { revisão sobre os } \\
\text { processos de } \\
\text { automação } \\
\text { laboratorial, bem } \\
\text { como sua utilização } \\
\text { em cada etapa do } \\
\text { fluxo de processos } \\
\text { em um laboratório } \\
\text { clínico demonstrando } \\
\text { sua relação com a } \\
\text { qualidade. }\end{array}$ & $\begin{array}{l}2011 \\
\text { Brasil }\end{array}$ & $\begin{array}{l}\text { J Bras Patol } \\
\text { Med Lab }\end{array}$ \\
\hline $\begin{array}{l}\text { Automation in Clinical } \\
\text { Biochemistry: Core, } \\
\text { Peripheral,STAT, and } \\
\text { Specialist Laboratories } \\
\text { in Australia. }\end{array}$ & $\begin{array}{l}\text { Streitberg, } \\
\text { G.S. et al. }\end{array}$ & $\begin{array}{l}\text { Estudo de caso, } \\
\text { foram enviados a } \\
\text { alguns laboratórios } \\
\text { questionários para } \\
\text { verificar informações } \\
\text { como localização e } \\
\text { tipo de laboratório, } \\
\text { carga de trabalho, } \\
\text { disponibilidade de } \\
\text { tecnologias, } \\
\text { procurando investigar } \\
\text { o papel da automação } \\
\text { em diversos } \\
\text { laboratórios e fez } \\
\text { parte de um grande } \\
\text { estudo sobre o } \\
\text { impacto da tecnologia } \\
\text { na formação e } \\
\text { recrutamento de } \\
\text { funcionários. }\end{array}$ & $\begin{array}{l}2012 \\
\text { Australia }\end{array}$ & $\begin{array}{l}\text { Journal of } \\
\text { Laboratory } \\
\text { Automation }\end{array}$ \\
\hline $\begin{array}{l}\text { Clinical Chemistry } \\
\text { Laboratory Automation } \\
\text { in the } 21 \text { st Century }\end{array}$ & $\begin{array}{l}\text { Armbruster } \\
\text { et al. }\end{array}$ & $\begin{array}{l}\text { Revisão literária da } \\
\text { automação } \\
\text { laboratorial, } \\
\text { descrevendo os } \\
\text { pontos mais } \\
\text { relevantes e } \\
\text { demonstrando o } \\
\text { processo de } \\
\text { automação } \\
\text { laboratorial total e } \\
\text { como isso facilitou o } \\
\text { diagnóstico. }\end{array}$ & $\begin{array}{l}2014 \\
\text { EUA }\end{array}$ & $\begin{array}{l}\text { Clin Biochem } \\
\text { Ver }\end{array}$ \\
\hline $\begin{array}{l}\text { The use of indicators in } \\
\text { the pre-analytical } \\
\text { phase as a laboratory } \\
\text { management tool }\end{array}$ & $\begin{array}{l}\text { Codagnone, } \\
\text { F.T. et al. }\end{array}$ & $\begin{array}{l}\text { Estudo de caso, } \\
\text { avaliou a frequência } \\
\text { de erros na fase pré- } \\
\text { analítica do } \\
\text { laboratório clínico de } \\
\text { um hospital militar. }\end{array}$ & $\begin{array}{l}2014 \\
\text { Brasil }\end{array}$ & $\begin{array}{l}\text { J Bras Patol } \\
\text { Med Lab }\end{array}$ \\
\hline $\begin{array}{l}\text { The add value of } \\
\text { laboratory diagnostics: } \\
\text { the many reasons why } \\
\text { decision-makers }\end{array}$ & $\begin{array}{l}\text { Lippi G, } \\
\text { Plebani M. }\end{array}$ & $\begin{array}{l}\text { Visa apresentar } \\
\text { elementos que } \\
\text { demonstrem que a } \\
\text { atividade dos }\end{array}$ & $\begin{array}{l}2017 \\
\text { Itália }\end{array}$ & $\begin{array}{l}\text { J Lab Precis } \\
\text { Med }\end{array}$ \\
\hline
\end{tabular}




\begin{tabular}{|ll|}
\hline should actually care & profissionais de \\
& laboratório está \\
& mudando, atualmente \\
& muito mais voltadas \\
& para gestão do que \\
& análises de amostras \\
& em si. Mostra que a \\
& medicina laboratorial \\
& é um ponto chave \\
& para um futuro de \\
& assistência à saúde \\
& centrada no paciente \\
& e sustentabilidade. \\
& Avaliar as mudanças 2018 \\
& que estão ocorrendo \\
Clinical laboratory: & nos serviços \\
bigger is not always & laboratoriais, que \\
better & variam desde \\
& aquisições à \\
& terceirização, \\
& principalmente com \\
& base nas expectativas \\
de melhorar a \\
eficiência, \\
aumentando os \\
volumes e reduzindo \\
os custos por teste. \\
\\
\end{tabular}

Dentre os textos encontrados na revisão, 5 deles ${ }^{(5,6,7,10,11)}$ apresentam aspectos de novas tecnologias e ferramentas de gestão da qualidade e seus impactos no desempenho de projetos na área da saúde, demonstrando que a automação laboratorial é uma forma tecnológica de expandir as competências de um laboratório, resultando em melhorias e confiabilidade maior de resultados. Assim como os métodos e equipamentos evoluíram com o passar do tempo a gestão da qualidade na área da saúde também precisou adequarse e criar programas que implementassem um processo permanente de melhoria da qualidade da assistência à saúde fazendo com que os serviços atingissem os padrões mais elevados de qualidade, surgiram então os programas de acreditação laboratorial, responsáveis por estabelecer padrões para realização de exames laboratoriais e promover uma melhoria continua da qualidade no setor.

Campana et al. descreve que a consolidação e integração entre as áreas de um laboratório geram redução dos custos e tendem a aumentar a utilização da automação pré-analítica. A estabilização de um número maior de tipos de ensaios e metodologias em equipamentos gera resultados importantes a nível de produtividade, diluição dos custos de insumos acessórios, menor manipulação de amostras, menor número de tubos por paciente e menor chance de erros, esta automação consiste na integração entre hardware e software desenhados para o processamento e análise por completo das amostras. Ressalta também que a evolução da automação laboratorial em 
todas as suas fases, está intimamente relacionada com a evolução em tecnologia da informação (TI). ${ }^{(4)}$

Os indicadores da qualidade no gerenciamento laboratorial auxiliam na padronização e definição das especificações da qualidade para o processo de realização de um exame, porém a grande variabilidade nos processos e fases dificultam o consenso da utilização de um em específico, atualmente a prática de benchmarking é uma alternativa muito aplicada, especialmente para comparar os indicadores mais conhecidos e utilizados. ${ }^{(7)}$

Os artigos da literatura científica demonstram que a organização do projeto é um ponto fundamental para que o sucesso planejado seja alcançado, acreditase que a escolha do melhor modelo de implementação dos processos é o ponto fundamental, baseando-se na realidade do laboratório e com o objetivo de reduzir os gastos e aumentar a qualidade e a objetividade dos serviços. ${ }^{(4,6)}$

A revisão inclui 3 textos ${ }^{(3,6,12)}$ que descrevem estudos de caso, demonstrando o impacto da tecnologia na administração e gestão de laboratórios na prática e seus resultados. Peck-Palmer demonstra a implementação de um sistema de automação num laboratório hospitalar e sua otimização após esta implementação. Os estudos demonstram em sua maioria que o processo de automação laboratorial inclui não apenas a implementação de tecnologias e equipamentos, mas também todos os eventos que ocorrerão após, que irão conectar todas as fases, pré-analítica, analítica e pós-analítica, os erros que acontecerão bem como as soluções encontradas e quais as ferramentas serão utilizadas para reportar esses dados e formar uma rede de informações com outras instituições e equipe multidisciplinar.

Plebani e Lippi descrevem o atual cenário laboratorial como promissor, os profissionais desta área após toda automação podem dedicar-se com maior disponibilidade a gestão da qualidade e todos os seus aspectos, uma vez que a automação possibilitou tempo hábil para tais tarefas e isto criou uma ampla rede de dados e permitiu que as instituições possam comunicar-se entre si, resultando em cuidados para saúde voltados para o paciente. Apresentam também que com o investimento em novas tecnologias, além de surgirem novas regulamentações e órgãos fiscalizadores que visam maior qualidade e segurança nos laboratórios, surge a necessidade de acompanhar os avanços tecnológicos e o monitoramento da qualidade por parte das instituições, sendo necessário que disponibilize subsídios para capacitação de profissionais, que possam atender aos desafios analíticos diários. ${ }^{(13,14)}$

\section{CONSIDERAÇÕES GERAIS}

O processo de automação laboratorial promoveu um avanço enorme no diagnóstico e prevenção de doenças, otimizando a eficiência dos laboratórios clínicos e garantindo segurança aos pacientes, atrelado a isso temos a expansão da gestão da qualidade, uma vez que os avanços tecnológicos requisitavam pessoal capacitado para transmitir os efeitos positivos deste 
avanço no setor, visando não apenas a geração de vantagens competitivas, mas que pudesse atender as necessidades da população.

Este estudo demonstra que a gestão da qualidade teve um papel muito importante no setor de automação laboratorial, uma vez que sem ela seria quase impossível alcançar o sucesso dos dias atuais, hoje implementar a automação em um laboratório de análises clínicas demanda tempo e inúmeros estudos por tratar-se de um setor de alta complexidade, porém existem profissionais capacitados que utilizam modelos de gestão e coordenação, promovendo compartilhamento de serviços aliados ao processo de sistematização, favorecendo não só a operação mas também o treinamento de equipes interdisciplinares contribuindo para o sucesso do projeto.

A implementação da automação laboratorial é uma tendência para os próximos anos, uma vez que é viável para diferentes modelos de empresas, empregando ferramentas e técnicas diversas para melhoria do fluxo de trabalho e que pode ser adequada nos pontos onde for mais necessária. O sucesso para qualquer processo de tomada de decisão está em buscar a melhor forma de alinhar a decisão à estratégia da instituição, com base em sua realidade, sempre com o objetivo de reduzir gastos e aumentar a qualidade e objetividade dos serviços, atendendo de forma integral as necessidades dos pacientes.

\section{REFERÊNCIAS}

1. Armbruster DA, Overcash DR, Reyes J. Clinical chemistry laboratory automation in the $21^{\text {st }}$ Century - amat victoria curam (victory loves careful preparation). Clin Biochem Rev. 2014;35(3):143-53. Disponível em: https://www.ncbi.nlm.nih.gov/pmc/articles/PMC4204236/pdf/cbr-35-143.pdf

2. Andriolo A. Breve história da automação em laboratórios clínicos. In: Recomendação da Sociedade Brasileira de Patologia Clínica/Medicina Laboratorial (SBPC/ML). Automação Laboratorial: histórico, seleção, implantação e gestão. São Paulo: Manole; 2018, P.1-30.

3. Streitberg GS, Angel L, Sikaris KA, Bwititi PT. Automation in clinical biochemistry: core, peripheral, STAT, and specialist laboratories in Australia. J Lab Autom. 2012;17(5): 387-94. DOI: 10.1177/2211068212448865

4. Campana GA, Oplustil CP, Faro LB. Tendências em medicina laboratorial. J Bras Patol Med Lab. 2011;47(4):399-408. Disponível em: https://www.redalyc.org/articulo.oa?id=393541961003

5. Campana GA, Oplustil CP. Conceitos de automação na medicina laboratorial: revisão de literatura. J Bras Patol Med Lab. 2011;47(2):119-27. Disponível em: https://www.redalyc.org/articulo.oa?id=393541959005

6. Codagnone FT, Alencar SMF, Shcolnik W, Chaves SRS, Silva LA, Henriques VHO, et al. The use of indicators in the pre-analytical phase as a 
laboratory management tool. J Bras Patol Med Lab. 2014;50(2):100-04.

DOI: http://dx.doi.org/10.5935/1676-2444.20140002

7. Vieira KF, Shitara ES, Mendes ME, Sumita NM. A utilidade dos indicadores da qualidade no gerenciamento de laboratórios clínicos. J Bras Patol Med Lab. 2011;47(3):201-10. Disponível em:

https://www.redalyc.org/articulo.oa?id=393541960002

8. Brasil. Ministério da Saúde. Agência Nacional de Vigilância Sanitária Resolução RDC n. 302, de 13 de outubro de 2005. Dispõe sobre requisitos para o funcionamento dos laboratórios clínicos e postos de coleta laboratorial públicos ou privados que realizam atividades na área de análises clínicas, patologia clínica e citologia.

9. Souza MT, Silva MD, Carvalho R. Revisão Integrativa: O que é e como fazer? São Paulo. 2010.

10. Trindade E. A incorporação de novas tecnologias nos serviços de saúde: o desafio da análise dos fatores em jogo. Cad Saúde Pública. 2008 24(5):95164. Disponível em: http://dx.doi.org/10.1590/S0102-311X2008000500002

11. Filho FUNB, Carvalho MMC, Ramos AW. Gerenciamento de projetos: o impacto do uso dos indicadores de desempenho no resultado do projeto. Prod e Produção. 2009;10(1):38-53. DOI: $\underline{10.22456 / 1983-8026.7786}$

12. Peck-Palmer O. Total lab automation takes teamwork. Med Lab Obs. 2009;41(10):30-34. Disponível em: https://www.researchgate.net/publication/38068742 Total lab automation t akes teamwork

13. Plebani M. Clinical laboratory: bigger is not always better. Diagnosis. 2018;5(2):41-46.DOI: https://doi.org/10.1515/dx-2018-0019.

14. Lippi G, Plebani M. The add value of laboratory diagnostics: the many reasons why decision-makers should actually care. J Lab Precis Med 2017;2(100):1-4. DOI: http://dx.doi.org/10.21037/jlpm.2017.12.07/

Recebido: 04 de setembro de 2019. Aceito: 10 de setembro de 2019

Correspondência: Bruna Ricelli. E-mail: brunaricelli16@gmail.com

Conflito de Interesses: os autores declararam não haver conflito de interesses. 
(C) This is an Open Access article distributed under the terms of the Creative Commons Attribution License, which permits unrestricted use, distribution, and reproduction in any medium, provided the original work is properly cited 